\title{
Safety of Liver Surface Instillation of Plasmid DNA in Normal and Carbon Tetrachloride-Induced Hepatitis Mice
}

\author{
Shintaro Fumoto, Hiroyuki Furukawa, Junzo Nakamura, Koyo Nishida \\ Graduate School of Biomedical Sciences, Nagasaki University, Nagasaki, Japan.
}

Received, April 19, 2011; Accepted, June 22, 2011; Published, June 27, 2011.

\begin{abstract}
Purpose. We previously demonstrated liver- and lobe-selective gene transfer following instillation of plasmid DNA (pDNA) onto the liver surface in mice. Safety concerns must be resolved prior to future clinical use. Thus, we investigated safety of liver surface instillation of pDNA in normal and hepatitis mice. Methods. pDNA was instilled onto the liver surface in normal and carbon tetrachloride-induced hepatitis mice. Gene expression (luciferase activity) and serum transaminase activities were measured at appropriate time points. Results. Transfection efficiency and target site selectivity were almost the same between the instillation of pDNA using a micropipette and a catheter. Serum transaminase activities $6 \mathrm{~h}$ after instillation of pDNA or a vehicle treatment using a micropipette were significantly higher than the no treatment mice, whereas instillation using a catheter did not raise serum transaminase activities throughout the tested time points, suggesting the safety of instillation using a catheter. This safety was also confirmed in hepatitis mice. A dose escalation study verified that liver surface instillation using a catheter did not raise serum transaminase at high doses with saturation of gene expression not only in normal mice, but also in hepatitis mice, supporting the safety of this method. Conclusions. We demonstrated that liver surface instillation of pDNA using a catheter did not raise serum transaminase activities. Information in this study will be useful for future clinical use of liver surface instillation of pDNA using a catheter.
\end{abstract}

This article is open to POST-PUBLICATION REVIEW. Registered readers (see "For Readers") may comment by clicking on ABSTRACT on the issue's contents page.

\section{INTRODUCTION}

Gene therapy is a promising approach for the treatment of acquired, refractory and fatal diseases as well as inherited gene deficiency diseases (1-5). Although enthusiastic investigations have been performed, clinical application of gene therapy is still limited. Major problems in the development of clinically applicable gene delivery system are efficacy and safety. Both viral and non-viral vectors have toxic side effects (6-9). There have been several attempts at reducing toxicity. Targeting of the foreign gene to a specific organ/tissue is rationalized strategy considering not only safety, but also efficacy. Receptor-mediated gene targeting appears to be a promising approach to obtain organ (or cell)-selective gene transfection (10). When foreign genes such as plasmid DNA (pDNA) were administered via the vasculature route, however, they were distributed to the whole body through the bloodstream, leading to inadequate organ-selective or diseased site-selective gene delivery, and were rapidly degraded by reticuloendothelial cells (liver Kupffer cells, etc.) and nuclease in the blood $(11,12)$. Moreover, both cationic liposomes and polymer/pDNA complex induces hemagglutination $(13,14)$. Therefore, safety concern along with gene delivery system must be resolved prior to clinical use.

Transfection utilizing naked pDNA is the simplest and safest non-viral gene delivery system since naked pDNA can be used without concerns about cytotoxicity by the gene carrier. Although it was previously reported that organ-selective gene transfection using naked pDNA was achieved by direct injection, electroporation, etc. (15-17), there is great concern about safety because these procedures require physical force against the organs; consequently, the continuous administration of pDNA is limited. On the other hand, we reported on organ- and site- selective gene expression following the instillation of naked pDNA to the liver surface $(18,19)$, kidney surface $(20)$, spleen surface (21), and gastric serosal surface (22) in mice. Organ surface instillation of naked pDNA does not require carriers and physical force to efficiently transfect the cells in vivo.

Corresponding Author: Shintaro Fumoto; Graduate School of Biomedical Sciences, Nagasaki University, 1-14 Bunkyo-machi, Nagasaki 852-8521, Japan; E-mail: sfumoto@nagasaki-u.ac.jp 
However, pDNA contains CpG motif, which induces production of proinflammatory cytokines $(23$, 24). In vivo gene transfer following the intravenous administration of cationic liposome/pDNA complex enhances serum inflammatory cytokine levels; as a consequence it causes elevated serum transaminase activities, i.e. liver damage $(8,9)$. Such safety concerns must be resolved before future clinical application. To confirm safety of our original method which simply instill naked pDNA onto the liver surface, in this study, serum transaminase activities after administration of pDNA were evaluated in mice. Moreover, on the assumption that the instillation of naked pDNA onto the liver surface will be applied to hepatitis patients, it was also tested whether this method deteriorates hepatitis induced by carbon tetrachloride in mice.

\section{MATERIALS AND METHODS}

\section{Materials}

Carbon tetrachloride was purchased from Nacalai Tesque, Inc. (Kyoto, Japan). All other chemicals were of the highest purity available.

\section{Animals}

Male ddY mice were housed in a cage in an air-conditioned room and maintained on a standard laboratory diet (MF, Oriental Yeast, Co., Ltd., Tokyo, Japan) and water ad libitum. All animal experiments were carried out in accordance with the Principles of Laboratory Animal Care as adopted and promulgated by the US National Institutes of Health and the Guidelines for Animal Experimentation of Nagasaki University.

\section{Preparation of pDNA}

pDNA (pCMV-luciferase) encoding firefly luciferase was constructed previously (18). pDNA was amplified in the Escherichia coli (E. coli) strain DH5a, isolated, and purified using an EndoFree ${ }^{\circledR}$ Plasmid Giga Kit (QIAGEN GmbH, Hilden, Germany). pDNA dissolved in phosphate-buffered saline solution was stored at $-20^{\circ} \mathrm{C}$ prior to experiments.

\section{In Vivo Gene Expression Experiments}

Five-week-old male ddY mice (19-38 g) were anesthetized with sodium pentobarbital $(40-60 \mathrm{mg} / \mathrm{kg}$, intraperitoneal injection). As conventional experiments, the middle abdomen was cut open and pDNA solution was instilled onto the surface of the liver left lateral lobe using micropipette (PIPETMAN ${ }^{\circledR}$, GILSON, Villier-le Bel, France). In case of the catheter, the middle abdominal skin was cut open, catheter (polyethylene tubing, PE10, Becton Dickinson and Company, MD, USA) with microsyringe (Hamilton, Bonaduz, Switzerland) was inserted into the pinhole at abdominal wall near from liver left lateral lobe, and pDNA solution was instilled onto the surface of the liver left lateral lobe. After administration of pDNA, the abdominal wall and skin was sutured, and then mice were freed in the cage. At appropriate time intervals, blood was collected, and the mice were killed under anesthesia, and the liver was removed. Then, the applied (left lateral) liver lobe was separated from other liver lobes. The liver samples were washed twice with saline and homogenized with a lysis buffer which consisted of $0.1 \mathrm{M}$ Tris/HCl buffer ( $\mathrm{pH} 7.8$ ) containing $0.05 \%$ Triton X-100 and 2 mM EDTA. The volumes of the lysis buffer added were $4 \mu \mathrm{l} / \mathrm{mg}$ for liver samples. The homogenates were centrifuged at $15,000 \times g$ for 5 min. Twenty microliters of supernatant was mixed with $100 \mu \mathrm{l}$ of luciferase assay substrates (PicaGene ${ }^{\circledR}$, Toyo Ink Mfg. Co., Ltd., Tokyo) and the light produced was immediately measured using a luminometer (MiniLumat LB 9506, BERTHOLD TECHNOLOGIES, Bad Wildbad, Germany). The luciferase activity is indicated as the relative light units (RLU) per gram of tissue.

\section{Determination of Serum Transaminase Activities}

Blood samples from in vivo gene expression experiments were clotted, centrifuged, and serum samples were obtained. Serum transaminase (GPT and GOT) activities were determined by POP-TOOS method using Transaminase C-II test Wako kit (Wako Pure Chemical Industries, Ltd., Osaka, Japan).

\section{Carbon Tetrachloride-Induced Hepatitis Mice}

Hepatitis mice were prepared by intraperitoneal injection of carbon tetrachloride in olive oil $(1 \%, 200$ $\mu \mathrm{l} / 20 \mathrm{~g}$ mice). Twenty four hours after carbon tetrachloride injection, pDNA was administered and the mice were subjected to gene expression experiment and measurement of serum transaminase activities as described above.

\section{STATISTICALANALYSIS}

Statistical comparisons were performed by Steel-Dwass non-parametric multiple comparison tests for gene expression, and by Dunnett parametric multiple comparison tests for serum transaminase activities. 


\section{RESULTS}

\section{Comparison between Conventional Liver Surface} Instillation and Instillation Using Catheter

Figure 1 shows time course of gene expression following the instillation of pDNA $(10 \mu \mathrm{g} / 5 \mu \mathrm{l})$ onto the liver surface using micropipette and catheter in mice. The gene expression levels in applied liver lobe by micropipette and catheter were comparable during indicated time points. Moreover, in both micropipette and catheter, gene expressions in applied liver lobe were significantly higher than those in other liver lobes except for $48 \mathrm{~h}$ in catheter group. Thus, transfection efficiency and target site selectivity were similar in the instillation of pDNA using micropipette and catheter. At the same moment, serum transaminase activities were also determined (Figure 2). In case of micropipette, serum GPT (Figure 2A) and GOT (Figure 2B) activities $6 \mathrm{~h}$ after pDNA administration were significantly higher than those in no treatment group, especially high value in GOT. However, GPT and GOT activities in vehicle group were also high, and they were comparable with pDNA group. Therefore, laparotomy for vehicle and pDNA treatment using micropipette might induce transient damage to mice. On the contrary, the instillation using catheter did not raise GPT and GOT activities during indicated time points, suggesting that this method did not induce liver damage.

\section{Safety of the Instillation of pDNA Using Catheter in Carbon Tetrachloride-Induced Hepatitis Mice}

There is a possibility that instillation of pDNA onto liver surface may deteriorate hepatitis when this method is applied for hepatitis patients. To test this possibility, the instillation of pDNA using catheter in carbon tetrachloride-induced hepatitis mice was evaluated (Figure 3 and 4). The gene expression in applied lobe of normal and hepatitis mice was comparable and significantly higher than that in other liver lobe, suggesting the liver lobe-selective gene transfer following the instillation of pDNA using catheter onto liver surface was also possible in hepatitis mice (Figure 3). Figure 4 shows serum transaminase activities after the instillation of pDNA using catheter onto liver surface in hepatitis mice. As of normal mice, serum GPT and GOT activities after the instillation using catheter in hepatitis mice were comparable with carbon tetrachloride-treated and vehicle groups. In addition, serum GPT and GOT activities $48 \mathrm{~h}$ after the instillation of pDNA using catheter were almost same as no treatment group.

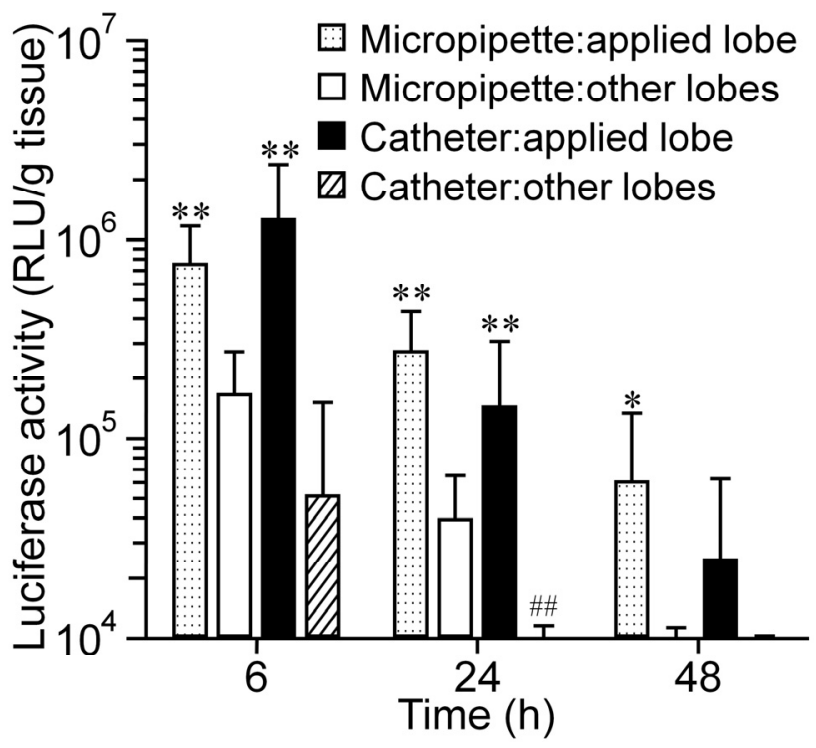

Figure 1. Comparison of gene expression between conventional liver surface instillation and instillation using catheter in mice. Gene expression in the applied lobe and other lobes of liver was determined 6, 24 and $48 \mathrm{~h}$ after naked pDNA administration (10 $\mu \mathrm{g} / 5 \mu \mathrm{l})$ using micropipette and catheter. The bar represents the mean + S.D. of at least eight experiments. Statistically significant differences compared with other lobes $\left({ }^{*} P<0.05,{ }^{* *} P<0.01\right)$ and with micropipette group $(\# P<0.01)$. 

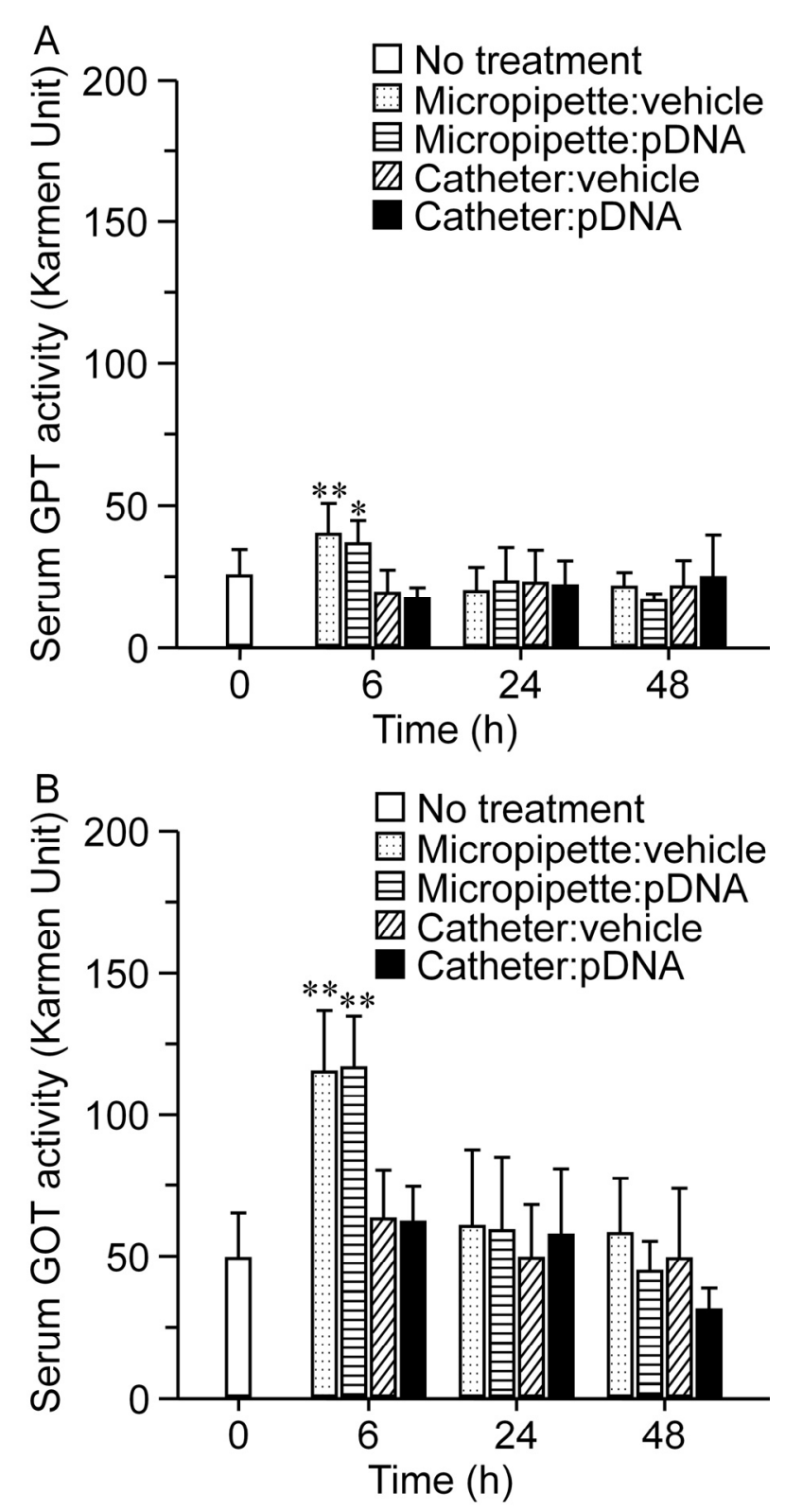

Figure 2. Comparison of serum GPT (A) and GOT activities (B) between conventional liver surface instillation and instillation using catheter in mice. Serum was collected 6,24 and $48 \mathrm{~h}$ after PBS (vehicle, $5 \mu \mathrm{l})$ or naked pDNA administration $(10 \mu \mathrm{g} / 5 \mu \mathrm{l})$, subsequently GPT and GOT activity was determined. The bar represents the mean + S.D. of at least eight experiments. Statistically significant differences compared with no treatment group $\left(* P<0.05,{ }^{* *} P<0.01\right)$.

\section{Dose Escalation Study}

To examine the safety of the instillation of pDNA using catheter further, pDNA dose was increased up to $60 \mu \mathrm{g}$ per mouse (Figure 5 and 6 ). The gene expression levels at doses of 30 and $60 \mu \mathrm{g}$ in both normal and hepatitis mice were comparable with those at a dose of $10 \mu \mathrm{g}$ (Figure 5), suggesting the saturation of gene expression was occurred as reported previously $(18,19)$. Figure 6 shows serum transaminase activities after the instillation of pDNA ( 30 and $60 \mu \mathrm{g}$ ) using catheter onto liver surface in normal and hepatitis mice. In both normal mice and hepatitis mice, the instillation of pDNA using catheter did not raise serum transaminase activities at high doses with saturation of gene expression. 


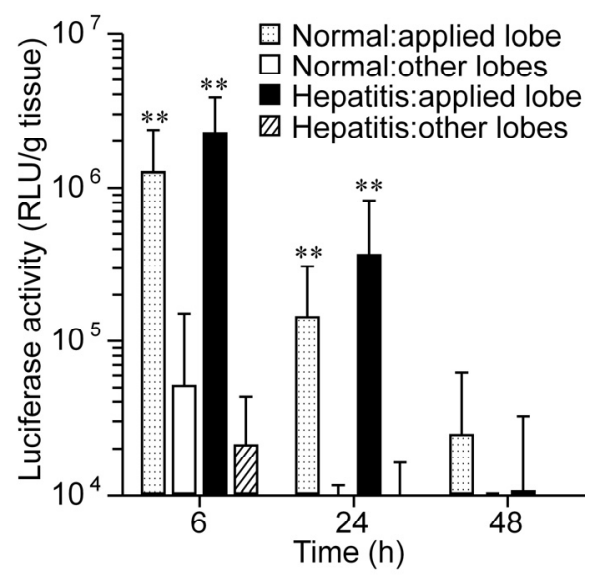

Figure 3. Comparison of gene expression in the liver between normal and carbon tetrachloride-induced hepatitis mice after liver surface instillation of naked pDNA using catheter. Gene expression in the applied lobe and other lobes was determined 6, 24 and $48 \mathrm{~h}$ after naked pDNA administration. The bar represents the mean + S.D. of at least eight experiments. Statistically significant differences compared with other lobes $(* * P<0.01)$. There were no significant differences between normal and hepatitis mice.
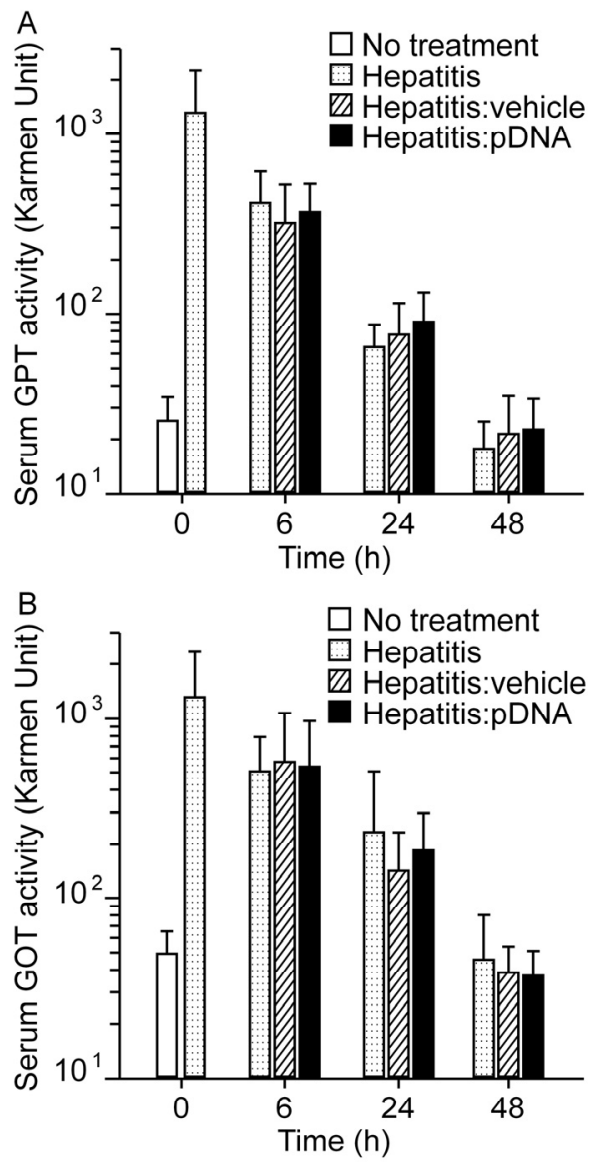

Figure 4. Serum GPT (A) and GOT activities (B) after liver surface instillation of pDNA using catheter in carbon tetrachloride-induced hepatitis mice. Serum was collected 6, 24 and $48 \mathrm{~h}$ after PBS (vehicle, $5 \mu \mathrm{l}$ ) or naked pDNA administration $(10 \mu \mathrm{g} / 5 \mu \mathrm{l})$, subsequently GPT and GOT activity was determined. The bar represents the mean + S.D. of at least eight experiments. There was no significant difference among hepatitis mice. 


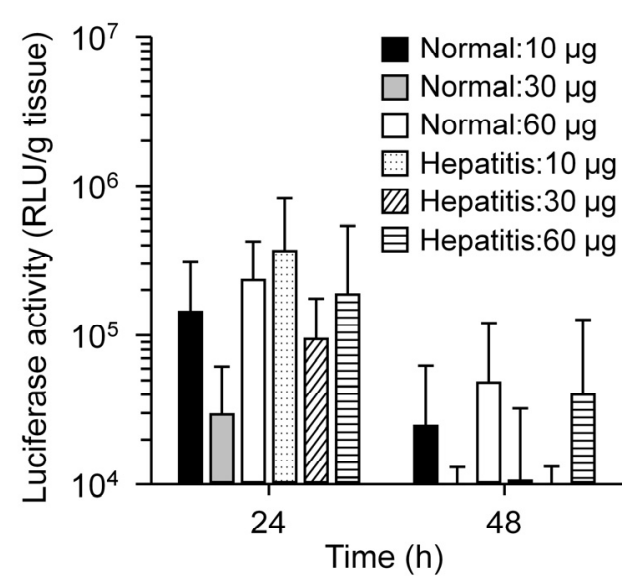

Figure 5. Effect of dose on gene expression in the applied liver lobe in normal and carbon tetrachloride-induced hepatitis mice after liver surface instillation of naked pDNA using catheter. Gene expression in the applied lobe was determined 24 and $48 \mathrm{~h}$ after naked pDNA administration $(10 \mu \mathrm{g} / 5 \mu \mathrm{l}, 30 \mu \mathrm{g} / 15 \mu \mathrm{l}$ or $60 \mu \mathrm{g} / 30 \mu \mathrm{l})$. The bar represents the mean + S.D. of at least seven experiments. There were no significant differences.
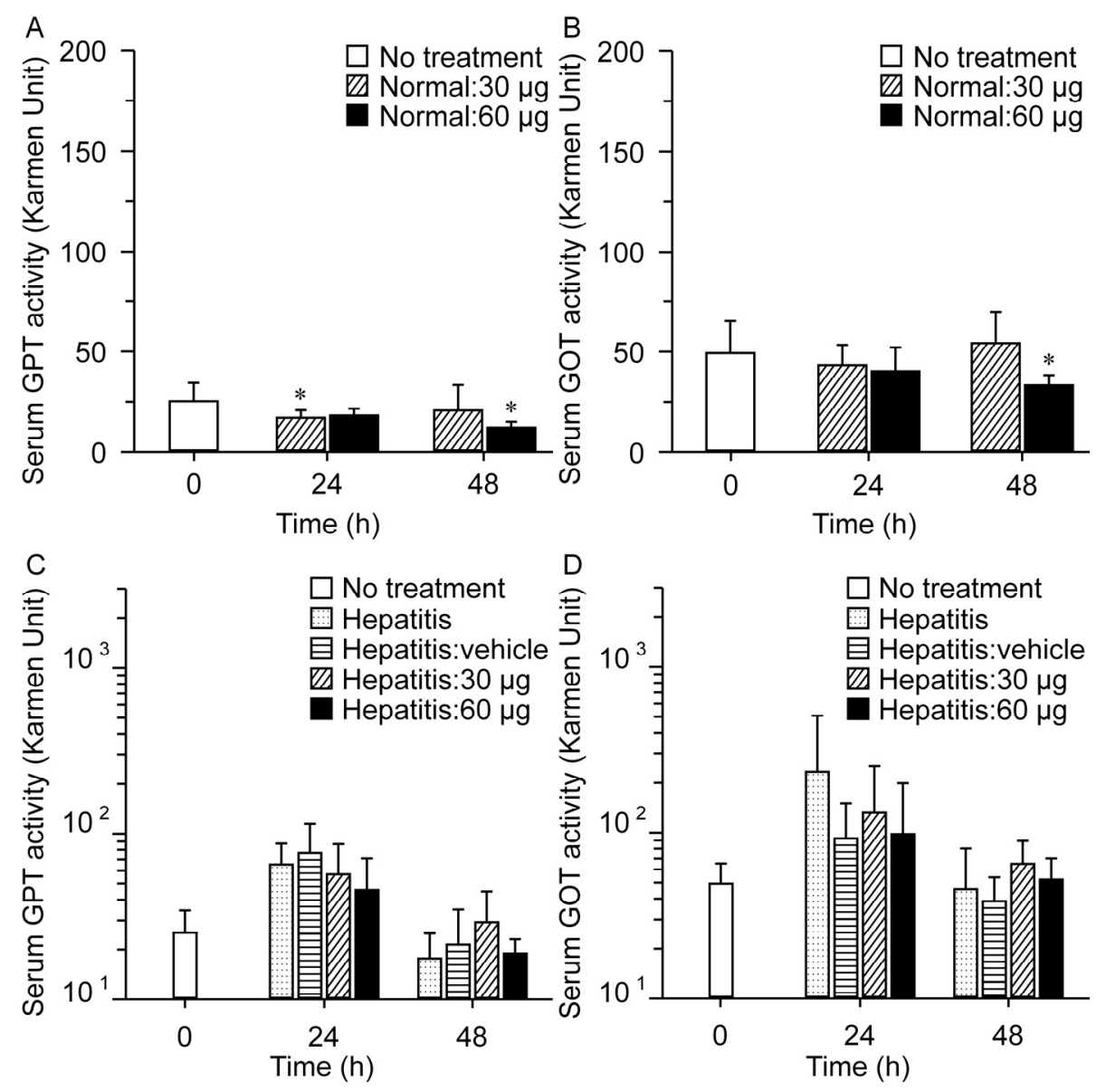

Figure 6. Effect of dose on serum GPT $(A, C)$ and GOT activities $(B, D)$ after liver surface instillation of pDNA using catheter in normal (A, B) and carbon tetrachloride-induced hepatitis mice (C, D). Serum was collected 24 and 48 h after naked pDNA administration $(30 \mu \mathrm{g} / 15 \mu \mathrm{l}$ or $60 \mu \mathrm{g} / 30 \mu \mathrm{l})$, subsequently GPT and GOT activity was determined. The bar represents the mean + S.D. of at least six experiments. (A, B) Statistically significant differences compared with no treatment mice $\left({ }^{*} P<0.05\right)$. (C, D) There were no significant differences between hepatitis mice and instillation mice. 


\section{DISCUSSION}

The liver plays an essential role in metabolism and excretion of endogenous and exogenous substances. Moreover, it is responsible for the synthesis of a wide variety of proteins that play important physiological roles. Liver diseases such as acute and chronic hepatitis, hepatoma, as well as inheritable gene deficiency diseases, are serious problems. Gene transfer to the liver is of great therapeutic potential for these refractory and fatal diseases. Furthermore, gene transfer such as a hepatocyte growth factor (HGF) for donor and recipient of live donor liver transplantation is a rationalized strategy for liver regeneration. However, gene expression in non-target sites would result in toxic side effect since HGF and other therapeutic proteins have high biological activity. Therefore, target site selectivity of gene expression is considered as an important factor for successful gene therapy. We have developed novel gene delivery system which transfer foreign gene selectively to the target organ/site by instilling naked pDNA onto the target site in mice (18-22).

Taking safety into account, however, not only side effects of expressed proteins, but also toxicity of vector itself, should be considered. Viral vectors have immunogenicity; consequently repeated administration is generally limited. Some viral vectors randomly insert foreign gene into genome, as a result, they have tumorigenicity. On the other hand, non-viral vectors such as cationic liposomes and polymers have also toxic side effect. Cationic liposome/pDNA complex and polyethyleneimine/pDNA complex induces hemagglutination $(13,14)$. Cationic liposomes induce apoptosis of macrophages (25). Furthermore, pDNA generally contains $\mathrm{CpG}$ motif (26), and induces inflammatory response by such as macrophages (27). As a consequence, cationic liposome/pDNA complex induces liver damage $(8,9)$. In case of liver surface instillation of naked pDNA, there is a possibility that peritoneal macrophage may take up pDNA and subsequently may induce inflammation. To evaluate this possibility, serum transaminase activities were measured in this study.

Conventional liver surface instillation of pDNA using a micropipette raised serum GPT and GOT activities transiently. However, vehicle treatment also raised serum GPT and GOT activities. In addition, increase of serum GOT activity was more remarkable. It is known that GOT is contained in not only hepatocytes, but also other cells such as myocytes. Therefore, this result might be due to cutting of abdominal wall, not due to liver damage. In contrast, liver surface instillation using catheter did not raise serum GPT and GOT activities. The difference between a micropipette and catheter may be attributed to the surgical operation, since only pinhole on abdominal wall was made in the case of catheter. Moreover, in hepatitis mice, the instillation using catheter also did not raise serum transaminase. In future clinical trial, gene therapy will be performed in hepatitis patients. Thus, safety information in not only normal but also hepatitis mice is important. Dose escalation study demonstrated that liver surface instillation using catheter did not raise serum transaminase at high doses with saturation of gene expression not only in normal mice but also in hepatitis mice, supporting the safety of this method. Gene expression after instillation of pDNA onto the liver surface was transient both in normal and hepatitis mice, thus repeated administration might be required for successful gene therapy. Safety in repeated administration is currently unclear, so it should be determined in the further study.

Proinflammatory cytokines were not measured, but there is a possibility that small amounts of them may be produced by liver surface instillation of pDNA. If production of proinflammatory cytokines were enhanced by liver surface instillation of pDNA in hepatitis mice, hepatitis would be deteriorated. However, liver surface instillation of pDNA in hepatitis mice did not deteriorate hepatitis. In addition, intravenous injection of naked pDNA does not induce production of proinflammatory cytokines (28). Thus the influence of this possibility might be negligible. On the other hand, proinflammatory cytokines, such as IFN- $\gamma$ and TNF- $\alpha$, cause inhibition of gene expression after intravenous injection of cationic lipidic vectors (29). This inhibition of gene expression is recovered by dexamethasone treatment (30). In case of gene transfer in hepatitis patients, concomitant administration of drugs such as dexamethasone for treatment of hepatitis is expected in the future clinical use. Therefore, effects of these drugs on gene expression should be investigated in the further study.

In this study, lipopolysaccharide-removal kit was used for purification of pDNA from $E$. coli lysate. Contamination of lipopolysaccharide in pDNA solution may activate peritoneal macrophages. It was reported that further purification of pDNA with Triton $\mathrm{X}-114$ resulted in restricted cytokine production from peritoneal macrophages in vitro (31). Thus this purification would improve safety of gene transfer using pDNA. 
In conclusion, we demonstrated that use of catheter for liver surface instillation of naked pDNA increased safety; as a consequence, serum transaminase activities after liver surface instillation of naked pDNA were kept at normal level in mice. Moreover, liver surface instillation of naked pDNA using catheter did not deteriorate carbon tetrachloride-induced hepatitis in mice, suggesting naked pDNA transfer could perform even in hepatitis condition. Important points for high safety were use of catheter and use of naked pDNA. Especially, naked pDNA transfer did not require both cationic carriers and physical forces causing toxic side effects. Information in this study will be useful for future clinical use of liver surface instillation of pDNA using catheter.

\section{ACKNOWLEDGMENTS}

This work was supported in part by a Grant-in-Aid for Scientific Research from the Ministry of Education, Culture, Sports, Science and Technology, Japan and by a Grant-in-Aid for Scientific Research from the President of Nagasaki University.

\section{REFERENCES}

1. Karamouzis MV, Argiris A, Grandis JR. Clinical applications of gene therapy in head and neck cancer. Curr Gene Ther, 2007; 7:446-457.

2. Yokoo T, Sakurai K, Ohashi T, Kawamura T. Stem cell gene therapy for chronic renal failure. Curr Gene Ther, 2003; 3:387-394.

3. Zenclussen AC, Zenclussen ML, Ritter T, Volk HD. The use of gene therapy tools in reproductive immunology research. Curr Gene Ther, 2005; 5:459-466.

4. Sands MS, Davidson BL. Gene therapy for lysosomal storage diseases. Mol Ther, 2006; 13:839-849.

5. Buch PK, MacLaren RE, Ali RR. Neuroprotective gene therapy for the treatment of inherited retinal degeneration. Curr Gene Ther, 2007; 7:434-445.

6. Marshall E. Gene therapy death prompts review of adenovirus vector. Science, 1999; 286:2244-2245.

7. Check E. A tragic setback. Nature, 2002; 420:116-118.

8. Tousignant JD, Gates AL, Ingram LA, et al. Comprehensive analysis of the acute toxicities induced by systemic administration of cationic lipid:plasmid DNA complexes in mice. Hum Gene Ther, 2000; 11:2493-2513.

9. Loisel S, Le Gall C, Doucet L, Ferec C, Floch V. Contribution of plasmid DNA to hepatotoxicity after systemic administration of lipoplexes. Hum Gene Ther, 2001; 12:685-696.

10. Kawakami S, Higuchi Y, Hashida M. Nonviral approaches for targeted delivery of plasmid DNA and oligonucleotide. J Pharm Sci, 2008; 97:726-745.

11. Kawabata K, Takakura Y, Hashida M. The fate of plasmid DNA after intravenous injection in mice: involvement of scavenger receptors in its hepatic uptake. Pharm Res, 1995; 12:825-830.

12. Mahato RI, Kawabata K, Nomura T, Takakura Y, Hashida M. Physicochemical and pharmacokinetic characteristics of plasmid DNA/cationic liposome complexes. J Pharm Sci, 1995; 84:1267-1271.

13. Eliyahu H, Servel N, Domb AJ, Barenholz Y. Lipoplex-induced hemagglutination: potential involvement in intravenous gene delivery. Gene Ther, 2002; 9:850-858.

14. Kircheis R, Wightman L, Schreiber A, et al. Polyethylenimine/DNA complexes shielded by transferrin target gene expression to tumors after systemic application. Gene Ther, 2001; 8:28-40.

15. Hickman MA, Malone RW, Lehmann-Bruinsma K, et al. Gene expression following direct injection of DNA into liver. Hum. Gene Ther, 1994; 5:1477-1483.

16. Somiari S, Glasspool-Malone J, Drabick JJ, et al. Theory and in vivo application of electroporative gene delivery. Mol Ther, 2000; 2:178-187.

17. Lin MTS, Pulkkinen L, Uitto J, Yoon K. The gene gun: current applications in cutaneous gene therapy. Int $\mathrm{J}$ Dermatol, 2000; 39:161-170.

18. Kawakami S, Hirayama R, Shoji K, et al. Liver- and lobe-selective gene transfection following the instillation of plasmid DNA to the liver surface in mice. Biochem Biophys Res Commun, 2002; 294:46-50.

19. Hirayama R, Kawakami S, Nishida K, et al. Development of the liver- and lobe-selective nonviral gene transfer following the instillation of naked plasmid DNA using catheter on the liver surface in mice. Pharm Res, 2003; 20:328-332.

20. Hirayama R, Nishida K, Fumoto S, Nakashima M, Sasaki H, Nakamura J. Unilateral kidney-selective gene transfer following the administration of naked plasmid DNA to the kidney surface in mice. Biol Pharm Bull, 2005; 28:181-184.

21. Nakamura J, Fumoto S, Kawanami R, et al. Spleen-selective gene transfer following the administration of naked plasmid DNA onto the spleen surface in mice. Biol Pharm Bull, 2007; 30:941-945.

22. Nishi J, Fumoto S, Ishii $\mathrm{H}$, et al. Improved stomach selectivity of gene expression following microinstillation of plasmid DNA onto the gastric serosal surface in mice. Eur J Pharm Biopharm, 2008; 69:633-639.

23. Krieg AM. Direct immunologic activities of CpG DNA implications for gene therapy. J Gene Med, 1999; 1:56-63.

24. Hemmi H, Takeuchi O, Kawai T, et al. A Toll-like receptor recognizes bacterial DNA. Nature, 2000; 408:740-745.

25. Aramaki Y, Takano S, Tsuchiya S. Induction of apoptosis in macrophages by cationic liposomes. FEBS Lett, 1999; 
460:472-476.

26. Niidome T, Huang L. Gene therapy progress and prospects: nonviral vectors. Gene Ther, 2002; 9:1647-1652.

27. Yamane I, Nishikawa M, Takakura Y. Cellular uptake and activation characteristics of naked plasmid DNA and its cationic liposome complex in human macrophages. Int J Pharm, 2005; 305:145-153.

28. Whitmore M, Li S, Huang L. LPD lipopolyplex initiates a potent cytokine response and inhibits tumor growth. Gene Ther, 1999; 6:1867-1875.

29. Li S, Wu SP, Whitmore M, et al. Effect of immune response on gene transfer to the lung via systemic administration of cationic lipidic vectors. Am J Physiol, 1999; 276:L796-L804.

30. Tan Y, Li S, Pitt BR, Huang L. The inhibitory role of CpG immunostimulatory motifs in cationic lipid vector-mediated transgene expression in vivo. Hum Gene Ther, 1999; 10:2153-2161.

31. Yasuda K, Kawano H, Yamane I, et al. Restricted cytokine production from mouse peritoneal macrophages in culture in spite of extensive uptake of plasmid DNA. Immunology, 2004; 111:282-290. 